\title{
THE FATOU COMPLETION OF A FRÉCHET FUNCTION SPACE AND APPLICATIONS
}

\author{
R. DEL CAMPO and W. J. RICKER ${ }^{\bowtie}$
}

(Received 7 November 2008; accepted 11 July 2009)

Communicated by A. J. Pryde

\begin{abstract}
Given a metrizable locally convex-solid Riesz space of measurable functions we provide a procedure to construct a minimal Fréchet (function) lattice containing it, called its Fatou completion. As an application, we obtain that the Fatou completion of the space $L^{1}(v)$ of integrable functions with respect to a Fréchetspace-valued measure $v$ is the space $L_{w}^{1}(v)$ of scalarly $v$-integrable functions. Further consequences are also given.
\end{abstract}

2000 Mathematics subject classification: primary 28B05; secondary 46A04, 46A40, 46E30, 54H12.

Keywords and phrases: Fréchet space (lattice), vector measure, Fatou property, Lebesgue topology, scalarly integrable function.

\section{Introduction}

Let $X$ be a Banach space and $v$ be a ( $\sigma$-additive) $X$-valued measure. Then the Banach space $L^{1}(v)$ of all $v$-integrable functions is a closed subspace (typically proper) of the Banach space $L_{w}^{1}(v)$ of all scalarly $v$-integrable functions [20]. Both $L^{1}(v)$ and $L_{w}^{1}(v)$ are Banach function spaces (relative to a control measure for $v$ and the pointwise almost everywhere order), with the distinction that $L^{1}(v)$ has order continuous norm (that is, a Lebesgue topology), whereas $L_{w}^{1}(v)$ always has the $\sigma$-Fatou property [6,7]. Actually, $L^{1}(v)$ is the order continuous part of $L_{w}^{1}(v)$, that is, the largest ideal inside $L_{w}^{1}(v)$ with each element having order continuous norm, and $L_{w}^{1}(v)$ is the $\sigma$-Fatou completion of $L^{1}(v)$, that is, the minimal Banach function space which has the $\sigma$-Fatou property and contains $L^{1}(v)$ [7].

What is the situation when $v$ takes its values in a Fréchet space $X$ ? It has been known for some time that $L^{1}(v)$ is complete, that is, is a Fréchet space [12]. In a recent article [8] it was shown that $L_{w}^{1}(v)$ is also complete and contains $L^{1}(v)$ as a

The first author thanks the Ministerio de Educación y Ciencia of Spain, for its support under the program 'José Castillejo'.

(c) 2009 Australian Mathematical Publishing Association Inc. 1446-7887/2009 \$16.00 
closed subspace. It is worth pointing out that the strong dual of a Fréchet space (or lattice or function space) is typically not a Fréchet space (or lattice or function space) and so certain duality arguments used in the Banach setting are not available in the Fréchet setting. It is also shown in [8] that both $L^{1}(v)$ and $L_{w}^{1}(v)$ are Fréchet function spaces with $L^{1}(v)$ having a Lebesgue topology, $L_{w}^{1}(v)$ having the $\sigma$-Fatou property and $L^{1}(v)$ being the order continuous part of $L_{w}^{1}(v)$. The aim of this note is to establish the 'missing link', namely, that $L_{w}^{1}(v)$ is the $\sigma$-Fatou completion of $L^{1}(v)$. In this regard, we develop various aspects of the theory of Fréchet function spaces (especially in relation to Lorentz function seminorms), which are not available in the literature in the form needed here; this, of interest in its own right, is done in Section 2. With these techniques we are able to establish close connections between $L^{1}(v)$ and $L_{w}^{1}(v)$ which are known in the Banach space setting [7]. Namely, in Section 3 it is shown that the following are equivalent: $L^{1}(v)=L_{w}^{1}(v) ; L^{1}(v)$ has the $\sigma$-Fatou property; $L_{w}^{1}(v)$ is order continuous; $L^{1}(\nu)$ is weakly sequentially complete; and $L_{w}^{1}(v)$ is weakly sequentially complete.

\section{Fréchet function spaces}

Let $(\Omega, \Sigma, \mu)$ be a $\sigma$-finite measure space and $\mathcal{M}:=L^{0}(\mu)$ be the space of all $\Sigma$-measurable, finite $\mathbb{R}$-valued $\mu$-a.e. functions on $\Omega$. Functions in $\mathcal{M}$ differing on a $\mu$-null set are identified. We define $\mathcal{M}^{+}:=\{f \in \mathcal{M} \mid f \geq 0\}$, where the notation $f \leq$ $g$ means that $f$ and $g$ are $\mathbb{R}$-valued and $f(x) \leq g(x)$ for $\mu$-a.e. $x \in \Omega$. According to [22, Ch. 15], a function seminorm in $\mathcal{M}$ is any function $\rho: \mathcal{M}^{+} \rightarrow[0, \infty]$ satisfying:

(i) $\quad \rho(u)=0$ if $u=0$ ( $\mu$-a.e.);

(ii) $\rho(a u)=a \rho(u)$ for all $a \geq 0$ and $u \in \mathcal{M}^{+}$;

(iii) $\rho(u+v) \leq \rho(u)+\rho(v)$ for all $u, v \in \mathcal{M}^{+}$;

(iv) $\rho(u) \leq \rho(v)$ whenever $u, v \in \mathcal{M}^{+}$satisfy $u \leq v$.

One can then extend $\rho$ to the whole of $\mathcal{M}$ (the extension is again denoted by $\rho$ ) by setting $\rho(f):=\rho(|f|)$, for any $f \in \mathcal{M}$. The closed unit ball $B_{\rho}:=\{f \in \mathcal{M} \mid \rho(f) \leq 1\}$ is solid, that is, if $f \in B_{\rho}$ and $g \in \mathcal{M}$ satisfy $|g| \leq|f|$, then also $g \in B_{\rho}$. If $\rho$ has the additional property that $\rho(f)=0$ if and only if $f=0$ ( $\mu$-a.e.), then it is called a function norm. In this case $L_{\rho}:=\{f \in \mathcal{M} \mid \rho(f)<\infty\}$ is a normed space (with $\rho$ as its norm) and is an ideal in $\mathcal{M}$, that is, $f \in \mathcal{M}$ and $g \in L_{\rho}$ with $|f| \leq|g|$ implies that $f \in L_{\rho}$ and $\rho(f) \leq \rho(g)$; see [22, Ch. 15] for all of these facts. Any normed space of this kind is called a Köthe function space and, if it is complete, a Banach function space.

Let $\left\{\rho_{n}\right\}_{n \in \mathbb{N}}$ be any increasing sequence of function seminorms in $\mathcal{M}$. We always assume that $\left\{\rho_{n}\right\}_{n \in \mathbb{N}}$ is fundamental, meaning that if $f \in \mathcal{M} \backslash\{0\}$, then there exists $m \in \mathbb{N}$ such that $\rho_{m}(f) \neq 0$. We then define

$$
L_{\left\{\rho_{n}\right\}}:=\left\{f \in \mathcal{M} \mid \rho_{n}(f)<\infty, \forall n \in \mathbb{N}\right\}=\bigcap_{n \in \mathbb{N}} L_{\rho_{n}} .
$$

The following fact is immediate from [15, Lemma 22.5]. For the notion of a locally solid topology we refer to [3, p. 33]. 
LEMMA 2.1. If $\left\{\rho_{n}\right\}_{n \in \mathbb{N}}$ is any increasing fundamental sequence of function seminorms, then $L_{\left\{\rho_{n}\right\}}$ is a metrizable, locally solid, locally convex Hausdorff space for the topology induced by $\left\{\rho_{n}\right\}_{n \in \mathbb{N}}$. Moreover, if $f \in \mathcal{M}$ and $g \in L_{\left\{\rho_{n}\right\}}$ satisfy $|f| \leq|g|$, then $f \in L_{\left\{\rho_{n}\right\}}$ and $\rho_{n}(f) \leq \rho_{n}(g)$ for all $n \in \mathbb{N}$.

Any locally convex Hausdorff space $L_{\left\{\rho_{n}\right\}}$ as given by Lemma 2.1 is called a (locally solid, metrizable) function space and, if complete, a Fréchet function space (F.f.s.).

Let $\Omega=[0,1]$ be equipped with Lebesgue measure $\mu$. For each $n \in \mathbb{N}$, let $A_{n}=$ $[0,1 / n]$ and define $\rho_{n}$ on $L^{0}(\mu)^{+}$by

$$
\rho_{n}(f):= \begin{cases}\int_{0}^{1 / n} f d \mu & \text { if } f \chi_{A_{n}}=f(\mu \text {-a.e. }) \\ \infty & \text { otherwise. }\end{cases}
$$

Then $\left\{\rho_{n}\right\}_{n \in \mathbb{N}}$ is an increasing fundamental sequence of function norms for which $L_{\left\{\rho_{n}\right\}}=\{0\}$. Let $\rho:=\rho_{2}$ be as above. Every Borel set $A \subseteq[1 / 2,1]$ with $\mu(A)>0$ satisfies $\rho\left(\chi_{A}\right)=\infty$, that is, [1/2,1] is a $\rho$-purely infinite set [22, Ch. 15, Section 67]. In this case, the carrier of the ideal $L_{\rho} \subseteq L^{0}(\mu)$ is not all of $\Omega$, but rather [0,1/2]. To avoid pathologies of the above kind, we call an increasing fundamental sequence of function seminorms $\left\{\rho_{n}\right\}_{n \in \mathbb{N}}$ determining if there exists $u \in L_{\left\{\rho_{n}\right\}}^{+}$satisfying $u>0$ pointwise $\mu$-a.e. on $\Omega$. It is useful to exhibit some examples in the nonnormable setting.

EXAMPLE 1. Let $X$ be a metrizable locally convex Hausdorff space generated by an increasing fundamental sequence of seminorms $\left(\|\cdot\|^{(n)}\right)_{n \in \mathbb{N}}$ in $X$ and with continuous dual space $X^{*}$. For each $n \in \mathbb{N}$, let

$$
\begin{gathered}
B_{n}:=\left\{x \in X:\|x\|^{(n)} \leq 1\right\}, \\
B_{n}^{\circ}:=\left\{x^{*} \in X^{*}:\left|\left\langle x, x^{*}\right\rangle\right| \leq 1, \forall x \in B_{n}\right\} .
\end{gathered}
$$

Given a ( $\sigma$-additive) vector measure $v: \Sigma \rightarrow X$, defined on a measurable space $(\Omega, \Sigma)$, a set $A \in \Sigma$ is called $\nu$-null if $\nu(B)=0$ for all $B \in \Sigma$ with $B \subseteq A$. Let $L^{0}(v)$ denote the Riesz space of all (equivalence classes modulo equality $v$-a.e. of) scalarvalued, $\Sigma$-measurable functions defined on $\Omega$, with respect to the $\nu$-a.e. pointwise order. For each $n \in \mathbb{N}$, define a $[0, \infty]$-valued seminorm $\|\cdot\|_{v}^{(n)}$ in $L^{0}(v)$ by

$$
\|f\|_{\nu}^{(n)}:=\sup _{x^{*} \in B_{n}^{\circ}} \int_{\Omega}|f| d\left|\left\langle\nu, x^{*}\right\rangle\right| \quad \forall f \in L^{0}(\nu),
$$

where $\left|\left\langle\nu, x^{*}\right\rangle\right|$ is the variation of the signed measure $\left\langle v, x^{*}\right\rangle: A \mapsto\left\langle v(A), x^{*}\right\rangle$ for all $x^{*} \in X^{*}$. If $\|f\|_{v}^{(n)}<\infty$ for all $n \in \mathbb{N}$, then $f$ is called scalarly $v$-integrable. This is equivalent to $\int_{\Omega}|f| d\left|\left\langle v, x^{*}\right\rangle\right|<\infty$ for all $x^{*} \in X^{*}$ [8, Proposition 2.1]. The space of all such functions $f \in L^{0}(v)$ is denoted by $L_{w}^{1}(v)$. Given a control measure $\mu$ for $v$ [12, pp. 19-21], we define the function seminorms

$$
\rho_{n}(f):=\|f\|_{v}^{(n)} \quad \forall f \in L^{0}(\mu)=L^{0}(v) \forall n \in \mathbb{N},
$$

in which case $L_{\left\{\rho_{n}\right\}}=L_{w}^{1}(v)$. So $L_{w}^{1}(v)$ is a F.f.s. [8, Theorem 2.5]. 
A function $f \in L_{w}^{1}(\nu)$ such that for each $A \in \Sigma$ there exists $\int_{A} f d \nu \in X$ satisfying $\left\langle\int_{A} f d v, x^{*}\right\rangle=\int_{A} f d\left\langle\nu, x^{*}\right\rangle$ for all $x^{*} \in X^{*}$ is called $\nu$-integrable. The subspace of $L_{w}^{1}(v)$ consisting of all $v$-integrable functions is denoted by $L^{1}(v)$. If $X$ is a Fréchet space and, for all $n \in \mathbb{N}$,

$$
\tilde{\rho}_{n}(f):= \begin{cases}\rho_{n}(f) & \text { if } f \in L^{1}(v) \\ \infty & \text { if } f \in L^{0}(\mu) \backslash L^{1}(v)=L^{0}(v) \backslash L^{1}(v)\end{cases}
$$

then $L_{\left\{\tilde{\rho}_{n}\right\}}=L^{1}(v)$ is also a F.f.s. [8, Theorem 2.5]. If $X$ is not complete, then the $\left\{\widetilde{\rho}_{n}\right\}_{n \in \mathbb{N}}$ are typically not function seminorms [17, Section 3]. Since the constant function $\chi_{\Omega}$ is positive $\mu$-a.e. and belongs to $L_{\left\{\rho_{n}\right\}}$ and to $L_{\left\{\tilde{\rho}_{n}\right\}}$, both $\left\{\rho_{n}\right\}_{n \in \mathbb{N}}$ and $\left\{\widetilde{\rho}_{n}\right\}_{n \in \mathbb{N}}$ are determining.

EXAMPLE 2. Let $\rho$ be any determining function norm in $L^{0}(\mu)$, with $(\Omega, \Sigma, \mu)$ a $\sigma$-finite, positive measure space. A Köthe matrix $A=\left(a_{n}\right)_{n}$ on $\Omega$ is any sequence of functions $a_{n} \in \mathcal{M}^{+}$which satisfy $0<a_{n} \leq a_{n+1}<\infty$ ( $\mu$-a.e. on $\left.\Omega\right)$, for each $n \in \mathbb{N}$. Define the normed Köthe function space $L_{\rho}\left(a_{n}\right):=\left\{f \in \mathcal{M} \mid \rho\left(a_{n} f\right)<\infty\right\}$, in which case $L_{\rho}\left(a_{n+1}\right) \subseteq L_{\rho}\left(a_{n}\right)$ and $L_{\left\{\rho_{n}\right\}}=\bigcap_{n=1}^{\infty} L_{\rho}\left(a_{n}\right)$ is a locally solid, metrizable function space, where $\rho_{n}(f):=\rho\left(a_{n} f\right), f \in \mathcal{M}$, for each $n \in \mathbb{N}$, is an increasing fundamental sequence of function norms. Such spaces have been treated in [4, 18], for example. If $\Omega$ is a countable set and $\mu$ is counting measure, then $L_{\left\{\rho_{n}\right\}}$ corresponds to a classical Köthe echelon space. For instance, with $\rho(x):=\left(\sum_{i \in \Omega}\left|x_{i}\right|^{p}\right)^{1 / p}$ and $p \in[1, \infty)$ fixed, such spaces are traditionally denoted by $\lambda_{p}(A)[15$, Ch. 27].

Further examples include $L_{p-}:=\bigcap_{1 \leq r<p} L^{r}([0,1])$ for $p \in(1, \infty)[5], \ell^{p+}:=$ $\bigcap_{p<q} \ell^{q}$ for $p \in[1, \infty)[16]$, and $L_{\text {loc }}^{p^{-}}(\mathbb{R})$, that is, $p$ th power locally integrable functions on $\mathbb{R}$ for $p \in[1, \infty)[1,2]$.

Let $(F, \tau)$ be a metrizable locally convex-solid Riesz space generated by a fundamental sequence of Riesz seminorms $\left\{q_{n}\right\}_{n \in \mathbb{N}}$ [3, Theorem 6.1]. Then $F$ has a Lebesgue ( $\sigma$-Lebesgue) topology if $u_{\alpha} \downarrow 0$ implies $u_{\alpha} \stackrel{\tau}{\rightarrow} 0$ in $F$ ( $u_{k} \downarrow 0$ implies that $u_{k} \stackrel{\tau}{\rightarrow} 0$ in $F$ ) [3, Ch. 3]. The space $F$ has the Fatou $(\sigma$-Fatou) property if, for every increasing net $\left(u_{\alpha}\right)_{\alpha}$ (increasing sequence $\left.\left(u_{k}\right)_{k}\right)$ in the positive cone $F^{+}$ of $F$ that is topologically bounded in $F$, the element $u:=\sup u_{\alpha}$ exists in $F^{+}$and $q_{n}\left(u_{\alpha}\right) \uparrow_{\alpha} q_{n}(u)\left(u:=\sup u_{k}\right.$ exists in $F^{+}$and $\left.q_{n}\left(u_{k}\right) \uparrow_{k} q_{n}(u)\right)$ for all $n \in \mathbb{N}$. This notion is not 'standard'; for example, in [3, p. 94] such a space $F$ is called a Nakano ( $\sigma$-Nakano) space.

An element $u$ of a Fréchet lattice $F$ is $\sigma$-order continuous if it has the property that $u_{k} \stackrel{\tau}{\rightarrow} 0$ as $k \rightarrow \infty$ for every sequence $\left(u_{k}\right)_{k} \subseteq F^{+}$satisfying $|u| \geq u_{k} \downarrow 0$. The $\sigma$-order continuous part $F_{a}$ of $F$ consists of the collection of all $\sigma$-order continuous elements of $F$; it is a closed ideal in $F$ [23, pp. 331-332], and clearly has a $\sigma$-Lebesgue topology.

If $F=L_{\left\{\rho_{n}\right\}}$ is a F.f.s., then there is no distinction between using nets and sequences for the Lebesgue and Fatou properties. Indeed, $L^{0}(\mu)$ is a $\sigma$-Dedekind complete Riesz 
space [14, pp. 126-127], and $F$ is an ideal in $L^{0}(\mu)$; see Lemma 2.1. So $F$ is also $\sigma$-Dedekind complete [14, Theorem 25.2]. Hence whenever $F$ has a $\sigma$-Lebesgue topology, it also has a Lebesgue topology [3, Theorem 17.9]. The converse is obvious. Concerning the Fatou and $\sigma$-Fatou properties, we need a preliminary result. For the notion of an order basis in a general Riesz space we refer to [14, Section 28] and for the concept of order separability we refer to [14, Section 23]. A normed lattice possessing these two properties has the Fatou property whenever it has the $\sigma$-Fatou property [23, Theorem 113.2]. The same is valid for Fréchet lattices.

PROPOSITION 2.2. Any order separable Fréchet lattice $F$ with a countable order basis and the $\sigma$-Fatou property has the Fatou property.

PROOF. Since $F$ has the $\sigma$-Fatou property, it follows from [14, Theorem 23.2(ii)] and [3, Theorem 5.4(i)] that $F$ is $\sigma$-Dedekind complete. By order separability, $F$ is then Dedekind complete [14, Theorem 23.6].

Let $\left\{b_{n}\right\}_{n \in \mathbb{N}}$ be an increasing, positive order basis of $F[14, \mathrm{p} .161]$. Consider a net $0 \leq u_{\lambda} \uparrow$ that is topologically bounded in $F$. We need to show that $\sup _{\lambda} u_{\lambda}$ exists in $F$ and that $q_{n}\left(\sup _{\lambda} u_{\lambda}\right)=\sup _{\lambda} q_{n}\left(u_{\lambda}\right)$ for all $n \in \mathbb{N}$. The proof proceeds as for normed spaces [23, Theorem 113.2]. Set $v_{\lambda, k}:=u_{\lambda} \wedge k b_{k}$ for all $\lambda$ and all $k \in \mathbb{N}$. For $k \in \mathbb{N}$ fixed, $v_{\lambda, k} \leq k b_{k}$ for all $\lambda$ and so $s_{k}:=\sup _{\lambda} v_{\lambda, k}$ exists in $F$ (by Dedekind completeness). Also, $s_{k}=\sup _{j} v_{\lambda_{j}, k}$ for some increasing sequence $\left(v_{\lambda_{j}, k}\right)_{j}$, by order separability of $F[14$, Theorem 23.2(iii)].

For all $k \in \mathbb{N}$, since $v_{\lambda_{j}, k} \uparrow_{j} s_{k}$, the $\sigma$-Fatou property of $F$ implies that $q_{n}\left(s_{k}\right)=$ $\sup _{j} q_{n}\left(v_{\lambda_{j}, k}\right)$ for all $n \in \mathbb{N}$. Hence $\left(s_{k}\right)_{k}$ is an increasing sequence that is topologically bounded in $F$ since, for all $n \in \mathbb{N}$,

$$
q_{n}\left(s_{k}\right)=\sup _{j} q_{n}\left(v_{\lambda_{j}, k}\right) \leq \sup _{j} q_{n}\left(u_{\lambda_{j}}\right) \leq \sup _{\lambda} q_{n}\left(u_{\lambda}\right)<\infty \quad \forall k \in \mathbb{N} .
$$

By the $\sigma$-Fatou property of $F$, it follows that $s:=\sup _{k} s_{k}$ exists in $F$ and that

$$
q_{n}(s)=\sup _{k} q_{n}\left(s_{k}\right) \leq \sup _{\lambda} q_{n}\left(u_{\lambda}\right) \quad \forall n \in \mathbb{N} .
$$

Since $\left\{b_{n}\right\}_{n \in \mathbb{N}}$ is an order basis, we have $s=\sup _{\lambda} u_{\lambda}$ [14, Theorem 28.2]. Thus $u_{\lambda} \uparrow s$. Moreover, $u_{\lambda} \leq s$ implies that $q_{n}\left(u_{\lambda}\right) \leq q_{n}(s)$ for all $n$ and $\lambda$ which, combined with (2.4), yields $q_{n}(s)=\sup _{\lambda} q_{n}\left(u_{\lambda}\right)$ for all $n \in \mathbb{N}$. So $F$ has the Fatou property.

COROLLARY 2.3. In every Fréchet function space, the $\sigma$-Fatou property and the Fatou property are equivalent.

PROOF. Let $L_{\left\{\rho_{n}\right\}}$ be a F.f.s. (over $(\Omega, \Sigma, \mu)$ ) with the $\sigma$-Fatou property. Note that $L^{0}(\mu)$ is a Dedekind complete, order separable Riesz space [14, pp. 126-127]. Since $L_{\left\{\rho_{n}\right\}}$ is an ideal in $L^{0}(\mu)$ (see Lemma 2.1) it follows that $L_{\left\{\rho_{n}\right\}}$ is order separable [10, Theorem 18.C]. Observe that $\chi_{\Omega} \in L^{0}(\mu)^{+}$satisfies $\sup _{k}\left(f \wedge k \chi_{\Omega}\right)=f$ for all $f \in L^{0}(\mu)$, and hence that $\left\{\chi_{\Omega}\right\}$ is a countable order basis of $L^{0}(\mu)$. Applying the last 
statement in [14, Theorem 29.3] to $L:=L^{0}(\mu)$, we see that the order separable ideal $L_{\left\{\rho_{n}\right\}}$ of $L^{0}(\mu)$ has a countable order basis. According to Proposition 2.2, $F$ has the Fatou property.

A function seminorm $\rho$ in $\mathcal{M}=L^{0}(\mu)$ has the Fatou property if $\rho\left(u_{k}\right) \uparrow \rho(u)$ whenever $0 \leq u_{k} \uparrow u$ in $\mathcal{M}$ [22, Ch. 15]. An increasing fundamental sequence of function seminorms $\left\{\rho_{n}\right\}_{n \in \mathbb{N}}$ is said to have the Fatou property if each $\rho_{n}$, for $n \in \mathbb{N}$, has it.

THEOREM 2.4. If $\left\{\rho_{n}\right\}_{n \in \mathbb{N}}$ is an increasing fundamental sequence of function seminorms with the Fatou property, then $L_{\left\{\rho_{n}\right\}}$ is a Fréchet function space with the Fatou property.

PROOF. Let $\left(u_{k}\right)_{k} \subseteq L_{\left\{\rho_{n}\right\}}^{+}$be increasing and topologically bounded. Fix $n_{0} \in \mathbb{N}$. Since $\rho_{n_{0}}$ is a function seminorm with the Fatou property and $\sup _{k} \rho_{n_{0}}\left(u_{k}\right)<\infty$, it follows from [22, Section 65, Theorem 3] that $\rho_{n_{0}}(u) \leq \sup _{k} \rho_{n_{0}}\left(u_{k}\right)<\infty$, where $u=\sup _{k} u_{k}=\lim _{k} u_{k}$ (pointwise). Hence $u \in L_{\left\{\rho_{n}\right\}}$. Moreover, $\rho_{n_{0}}\left(u_{k}\right) \uparrow \rho_{n_{0}}(u)$ as $\rho_{n_{0}}$ has the Fatou property as a function seminorm. This shows that $L_{\left\{\rho_{n}\right\}}$ has the $\sigma$-Fatou property. Thus, Corollary 2.3 and [3, Theorem 13.9] guarantee that $L_{\left\{\rho_{n}\right\}}$ is complete, that is, a F.f.s. with the Fatou property.

Recall that the Lorentz function seminorm $\rho_{L}$ associated with any function seminorm $\rho$ in $\mathcal{M}=L^{0}(\mu)$ is defined by

$$
\rho_{L}(u):=\inf \left\{\lim _{k} \rho\left(u_{k}\right) \mid u_{k} \in \mathcal{M}^{+}, u_{k} \uparrow u\right\} \quad \forall u \in \mathcal{M}^{+} ;
$$

it is the largest function seminorm with the Fatou property that is majorized by $\rho$ [22, Ch. 15, Section 66]. The following property of $\rho_{L}$ is established in the proof of Theorem 2 in [22, pp. 450-451].

FACT 1 . Given $u \in \mathcal{M}^{+}$, there exists a sequence $\left(u_{k}\right)_{k} \subseteq \mathcal{M}^{+}$satisfying $u_{k} \uparrow u$ and $\rho\left(u_{k}\right) \uparrow \rho_{L}(u)$.

LEMMA 2.5. Let $\left\{\rho_{n}\right\}_{n \in \mathbb{N}}$ be an increasing sequence of function seminorms in $L^{0}(\mu)$. Then also $\left\{\left(\rho_{n}\right)_{L}\right\}_{n \in \mathbb{N}}$ is an increasing sequence of function seminorms. Moreover, $\left\{\rho_{n}\right\}_{n \in \mathbb{N}}$ is fundamental if and only if $\left\{\left(\rho_{n}\right)_{L}\right\}_{n \in \mathbb{N}}$ is fundamental.

PROOF. The first statement follows from (2.5).

Concerning the second statement, suppose that $\left\{\left(\rho_{n}\right)_{L}\right\}$ is fundamental. Then it follows from $\left(\rho_{n}\right)_{L} \leq \rho_{n}$ that $\left\{\rho_{n}\right\}_{n \in \mathbb{N}}$ is also fundamental.

Conversely, assume that $\left\{\rho_{n}\right\}$ is fundamental. Suppose that there exists $0<u \in$ $L^{0}(\mu)^{+}$satisfying $\left(\rho_{n}\right)_{L}(u)=0$ for all $n \in \mathbb{N}$. Now, there is some $r>0$ and a subset $A \in \Sigma$ of $\{w \in \Omega \mid u(w)>r\} \in \Sigma$ such that $0<\mu(A)<\infty$. Since $r \chi_{A} \leq u$, we have $\left(\rho_{n}\right)_{L}\left(\chi_{A}\right)=0$ for all $n \in \mathbb{N}$. We proceed to construct a decreasing sequence $\left(A_{n}\right)_{n} \subseteq \Sigma$ satisfying $A_{n} \subseteq A$ with $\mu\left(A_{n}\right)>\frac{1}{2} \mu(A)$ and $\rho_{n}\left(\chi_{A_{n}}\right)=0$ for all $n \in \mathbb{N}$. 
For each $n \geq 2$, define $\alpha_{n}:=\left(1+2^{n}\right) /\left(2+2^{n}\right)$ and $\beta_{n}:=\frac{1}{2}+2^{-n}$. Observe that $\frac{1}{2}<\alpha_{n}<\alpha_{n+1}<1$ with $\alpha_{n} \uparrow 1$ and that $\frac{1}{2}<\beta_{n+1}<\beta_{n}<1$ with $\beta_{n} \downarrow \frac{1}{2}$. Moreover, $\alpha_{n} \beta_{n}=\beta_{n+1}>\frac{1}{2}$ for all $n \geq 2$.

Since $\left(\rho_{1}\right)_{L}\left(\chi_{A}\right)=0$, Fact 1 ensures for us the existence of a sequence $\left(u_{k}\right)_{k} \subseteq \mathcal{M}^{+}$ with $u_{k} \uparrow \chi_{A}$ and $\rho_{1}\left(u_{k}\right)=0$ for all $k \in \mathbb{N}$. Define $B_{k}:=\left\{w \in \Omega \mid u_{k}(w)>\frac{1}{2}\right\}$ for all $k \in \mathbb{N}$, in which case $B_{k} \uparrow A$. Accordingly, $\mu\left(B_{k}\right) \uparrow \mu(A)$ and so there exists $k_{0}$ such that $\mu\left(B_{k_{0}}\right)>\beta_{2} \mu(A)$. Moreover, from $\frac{1}{2} \chi_{B_{k_{0}}} \leq u_{k_{0}} \chi_{B_{k_{0}}} \leq u_{k_{0}}$, we conclude that $\rho_{1}\left(\chi_{B_{k_{0}}}\right)=0$. Set $A_{1}:=B_{k_{0}} \subseteq A$ so that $\rho_{1}\left(\chi_{A_{1}}\right)=0$ and $\mu\left(A_{1}\right)>\beta_{2} \mu(A)$.

Observe that $\left(\rho_{2}\right)_{L}\left(\chi_{A_{1}}\right) \leq\left(\rho_{2}\right)_{L}\left(\chi_{A}\right)=0$. Repeat the process with $\rho_{1}$ and $\chi_{A}$ replaced by $\rho_{2}$ and $\chi_{A_{1}}$, to produce $A_{2} \subseteq A_{1}$ such that $\rho_{2}\left(\chi_{A_{2}}\right)=0$ and $\mu\left(A_{2}\right)>$ $\alpha_{2} \mu\left(A_{1}\right)$, that is, $\mu\left(A_{2}\right)>\alpha_{2} \beta_{2} \mu(A)=\beta_{3} \mu(A)$.

Note that $\left(\rho_{3}\right)_{L}\left(\chi_{A_{2}}\right) \leq\left(\rho_{3}\right)_{L}\left(\chi_{A}\right)=0$. Again repeat the process for $\rho_{3}$ and $\chi_{A_{2}}$ to produce $A_{3} \subseteq A_{2}$ such that $\rho_{3}\left(\chi_{A_{3}}\right)=0$ and $\mu\left(A_{3}\right)>\alpha_{3} \mu\left(A_{2}\right)$, that is, $\mu\left(A_{3}\right)>$ $\alpha_{3} \beta_{3} \mu(A)=\beta_{4} \mu(A)$.

Proceed inductively so that at stage $n$ one produces $A_{n} \subseteq A_{n-1}$ such that $\rho_{n}\left(\chi_{A_{n}}\right)=0$ and $\mu\left(A_{n}\right)>\alpha_{n} \mu\left(A_{n-1}\right)$, that is, $\mu\left(A_{n}\right)>\alpha_{n} \beta_{n} \mu(A)=\beta_{n+1} \mu(A)>$ $\frac{1}{2} \mu(A)$. So the sequence of sets $\left(A_{n}\right)_{n}$ with the properties claimed above exists.

Finally, define $B:=\bigcap_{n=1}^{\infty} A_{n}$. Then

$$
\mu(B)=\lim _{n \rightarrow \infty} \mu\left(A_{n}\right) \geq \frac{1}{2} \mu(A)>0 .
$$

But $\rho_{n}\left(\chi_{B}\right) \leq \rho_{n}\left(\chi_{A_{n}}\right)=0$ for all $n \in \mathbb{N}$. Since $\left\{\rho_{n}\right\}$ is fundamental, it follows that $\chi_{B}=0$ in $L^{0}(\mu)$, that is, $\mu(B)=0$, which is a contradiction.

Let $\left\{\rho_{n}\right\}_{n \in \mathbb{N}}$ be any increasing fundamental sequence of function seminorms. By Lemma 2.5, the same is true of $\left\{\left(\rho_{n}\right)_{L}\right\}_{n \in \mathbb{N}}$. Hence Theorem 2.4 shows that $L_{\left\{\left(\rho_{n}\right)_{L}\right\}}=$ $\bigcap_{n \in \mathbb{N}} L_{\left(\rho_{n}\right)_{L}}$ is a F.f.s. with the Fatou property. The inequality $\left(\rho_{n}\right)_{L} \leq \rho_{n}$ for all $n \in \mathbb{N}$, implies that $L_{\left\{\rho_{n}\right\}} \subseteq L_{\left\{\left(\rho_{n}\right)_{L}\right\}}$ with a continuous inclusion. Moreover, given $n \in \mathbb{N}$, if $\rho$ is any function seminorm in $L^{0}(\mu)$ with the Fatou property such that $\left(\rho_{n}\right)_{L} \leq \rho \leq \rho_{n}$, then $\rho=\left(\rho_{n}\right)_{L}$ [22, Section 71, Theorems 2 and 3(d)]. Accordingly, $L_{\left\{\left(\rho_{n}\right)_{L}\right\}}$ is the minimal F.f.s. in $L^{0}(\mu)$ with the Fatou property and which contains $L_{\left\{\rho_{n}\right\}}$ continuously. By minimal we mean that if $\left\{\eta_{n}\right\}_{n \in \mathbb{N}}$ is any increasing fundamental sequence of function seminorms in $L^{0}(\mu)$ (for the same measure space $(\Omega, \Sigma, \mu)$ ) with the Fatou property and such that $\eta_{n} \leq \rho_{n}$ for all $n \in \mathbb{N}$, then $L_{\left\{\left(\rho_{n}\right)_{L}\right\}} \subseteq L_{\left\{\eta_{n}\right\}}$.

Definition 2.6. $L_{\left\{\left(\rho_{n}\right)_{L}\right\}}$ is called the Fatou completion of $L_{\left\{\rho_{n}\right\}}$ and is denoted by $\left(L_{\left\{\rho_{n}\right\}}\right)^{F}$.

\section{Applications}

As a first application of Theorem 2.4, observe that the completeness of $L_{w}^{1}(v)$ follows immediately since the function seminorms $\rho_{n}$ given by (2.2), which induce this space, obviously have the Fatou property by [22, Section 65, Theorem 4]. For an alternative proof see [8, Theorem 2.5]. 
It is important to note that $\left(L_{\left\{\rho_{n}\right\}}\right)^{F}$ is not necessarily obtained from $L_{\left\{\rho_{n}\right\}}$ in any topological sense. Indeed, if $X$ is a Banach space and $v$ is an $X$-valued vector measure, then for the (single) function norm

$$
\rho(f):= \begin{cases}\sup _{x^{*} \in B_{X^{*}}} \int_{\Omega}|f| d\left|\left\langle\nu, x^{*}\right\rangle\right| & \text { if } f \in L^{1}(v) \\ \infty & \text { if } f \in L^{0}(v) \backslash L^{1}(v),\end{cases}
$$

we have $L_{\rho}=L^{1}(v)$ whereas $\left(L_{\rho}\right)^{F}=L_{w}^{1}(v)$ [7]. For certain $v$, the space $L^{1}(v)$ can be a proper closed subspace of $L_{w}^{1}(\nu) ;[7],[12$, p. 31]. So in the Banach space setting we see that $L_{w}^{1}(v)$ is the Fatou completion of $L^{1}(v)$. We show that the same result holds in Fréchet spaces.

THEOREM 3.1. Let $v$ be any vector measure taking its values in a Fréchet space. Then the Fatou completion of $L^{1}(v)$ is precisely $L_{w}^{1}(v)$. Thus, $L^{1}(v)$ has the Fatou property if and only if $L^{1}(v)=L_{w}^{1}(v)$.

Proof. We use the notation of Example 1. Fix $n \in \mathbb{N}$. As noted above, $\rho_{n}$ has the Fatou property and, clearly, $\rho_{n} \leq \widetilde{\rho}_{n}$ in $L^{0}(\mu)$. By the maximal property of the Lorentz seminorm we conclude that $\rho_{n} \leq\left(\widetilde{\rho}_{n}\right)_{L}$ in $L^{0}(\mu)$. On the other hand, let $f \in L_{w}^{1}(v)^{+}$. Choose $\Sigma$-simple functions $0 \leq s_{k} \uparrow f$, in which case $\left(s_{k}\right)_{k} \subseteq L^{1}(v) \subseteq$ $L_{w}^{1}(v)$. By (2.5) applied to $\widetilde{\rho}_{n}$,

$$
\left(\widetilde{\rho}_{n}\right)_{L}(f) \leq \lim _{k} \widetilde{\rho}_{n}\left(s_{k}\right)=\lim _{k} \rho_{n}\left(s_{k}\right) \leq \rho_{n}(f),
$$

from which we can conclude that $\left(\widetilde{\rho}_{n}\right)_{L}(f)=\rho_{n}(f)$ for all $f \in L_{w}^{1}(v)$. These inequalities imply that $L_{w}^{1}(v)=L_{\left\{\rho_{n}\right\}} \subseteq L_{\left\{\left(\tilde{\rho}_{n}\right)_{L}\right\}}=\left(L^{1}(v)\right)^{F}$.

Let $f \in\left(L^{1}(v)\right)^{F}$, that is, $\left(\widetilde{\rho}_{n}\right)_{L}(f)<\infty$, for all $n \in \mathbb{N}$. Fix $m \in \mathbb{N}$. By Fact 1 applied to $\rho_{m}$ there exists $\left(u_{k}\right)_{k} \subseteq L^{0}(\mu)^{+}$satisfying $u_{k} \uparrow f$ with $\widetilde{\rho}_{m}\left(u_{k}\right) \uparrow\left(\widetilde{\rho}_{m}\right)_{L}(f)$. Then $\sup _{k} \widetilde{\rho}_{m}\left(u_{k}\right) \leq\left(\widetilde{\rho}_{m}\right)_{L}(f)<\infty$. From the definition of $\widetilde{\rho}_{m}$, we see that $\left(u_{k}\right)_{k} \subseteq$ $L^{1}(v) \subseteq L_{w}^{1}(v)$. Since $\rho_{m}$ has the Fatou property, we conclude that

$$
\rho_{m}(f)=\sup _{k} \rho_{m}\left(u_{k}\right)=\sup _{k} \widetilde{\rho}_{m}\left(u_{k}\right)<\infty .
$$

But $m \in \mathbb{N}$ is arbitrary and so $f \in L_{\left\{\rho_{n}\right\}}=L_{w}^{1}(v)$. This shows that $L_{w}^{1}(v)=\left(L^{1}(v)\right)^{F}$, with equality both as vector spaces and topologically.

If $L^{1}(v)=L_{w}^{1}(v)$, then $L^{1}(v)$ has the Fatou property. Conversely, assume that $L^{1}(v)$ has the Fatou property. It is always the case that $L^{1}(v) \subseteq L_{w}^{1}(v)$. Let $f \in L_{w}^{1}(v)^{+}$and choose $\Sigma$-simple functions $0 \leq f_{k} \uparrow f$. As $\left(f_{k}\right)_{k} \subseteq L^{1}(v)$, it follows from (2.2) and (2.3), for each $n \in \mathbb{N}$, that

$$
\tilde{\rho}_{n}\left(f_{k}\right)=\rho_{n}\left(f_{k}\right) \leq \rho_{n}(f)<\infty \quad \forall k \in \mathbb{N} .
$$

Hence $\left(f_{k}\right)_{k}$ is topologically bounded in $L^{1}(v)$. Since $L^{1}(v)$ has the Fatou property, it follows that $f=\sup _{k} f_{k} \in L^{1}(v)$. So $L^{1}(v)=L_{w}^{1}(v)$. 
For Banach spaces the above result is known [7], after observing that the second associate norm $\rho^{\prime \prime}$ of a function norm $\rho$ is precisely $\rho_{L}$ [22, p. 471].

The following result [8, Theorem 3.2], alluded to in the Introduction, is recorded for the sake of completeness.

THEOREM 3.2. For any vector measure $v$ taking values in a Fréchet space we have $\left(L_{w}^{1}(v)\right)_{a}=L^{1}(v)$. In particular, $L_{w}^{1}(v)$ has a Lebesgue topology if and only if $L_{w}^{1}(v)=L^{1}(v)$.

We provide further equivalent conditions for $L_{w}^{1}(v)=L^{1}(v)$ to hold.

A Fréchet lattice $F$ is called a KB-space if every topologically bounded, increasing sequence in $F^{+}$is convergent. If every topologically bounded, increasing sequence in $F^{+}$, with $F$ a Fréchet lattice, has a supremum in $F$, then $F$ is said to satisfy the $\sigma$-Levi property.

LEMMA 3.3. For any Fréchet lattice $F$ the following are equivalent.

(i) $F$ is a KB-space.

(ii) $F$ satisfies the $\sigma$-Levi property and has a $\sigma$-Lebesgue topology.

(iii) $F$ has a $\sigma$-Lebesgue topology and the $\sigma$-Fatou property.

(iv) $F$ contains no lattice copy of $c_{0}$.

(v) $F$ is weakly sequentially complete.

PROOF. The equivalence of (i) and (ii) is part of [9, Proposition 2.1].

To establish that (ii) implies (iii), let $\left\{q_{n}\right\}_{n \in \mathbb{N}}$ be a fundamental system of solid seminorms generating the topology $\tau$ of $F$. Let $\left(u_{k}\right)_{k} \subseteq F^{+}$be an increasing and topologically bounded sequence. By the $\sigma$-Levi property of $F, u=\sup _{k} u_{k}$ exists in $F^{+}$. Since (ii) implies (i), there exists $v \in F$ with $u_{k} \stackrel{\tau}{\rightarrow} v$. By [3, Theorem 5.6(iii)], $v=\sup _{k} u_{k}$, that is, $u=v$. Fix $n \in \mathbb{N}$. The inequality $\left|q_{n}\left(u_{k}\right)-q_{n}(u)\right| \leq q_{n}\left(u_{k}-u\right)$, for each $k \in \mathbb{N}$, shows that $q_{n}\left(u_{k}\right) \uparrow_{k} q_{n}(u)$.

That (iii) implies (ii) is obvious.

To establish that (iv) implies (ii), observe that the completeness of $F$ ensures the monotone completeness property (see [3, p. 45] for the definition). Then [9, Theorem 2.5] implies that $F$ satisfies the $\sigma$-Levi property and has a $\sigma$-Lebesgue topology.

To see that (ii) implies (iv), again note that the completeness of $F$ ensures the monotone completeness property. Then [9, Proposition 2.2 and Theorem 2.5] imply that $F$ cannot contain a lattice copy of $c_{0}$.

That (v) implies (iv) is clear. For if $F$ is weakly sequentially complete, then it cannot contain an isomorphic lattice copy of the Banach lattice $c_{0}[11,19]$.

Finally, that (iv) implies (v) follows from [21, Theorem 1].

For Banach spaces the following result occurs in [7].

PROPOSITION 3.4. Let $v$ be any vector measure taking values in a Fréchet space. Then the following statements are equivalent. 
(i) $L_{w}^{1}(v)=L^{1}(v)$.

(ii) $L_{w}^{1}(\nu)$ has a $\sigma$-Lebesgue topology.

(iii) $L^{1}(v)$ has the $\sigma$-Fatou property.

(iv) $L_{w}^{1}(v)$ is a $\mathrm{KB}$-space.

(v) $L^{1}(v)$ is a $\mathrm{KB}$-space.

(vi) $L_{w}^{1}(\nu)$ contains no lattice copy of $c_{0}$.

(vii) $L^{1}(v)$ contains no lattice copy of $c_{0}$.

(viii) $L_{w}^{1}(v)$ is weakly sequentially complete.

(ix) $L^{1}(v)$ is weakly sequentially complete.

PROOF. The equivalence of (i) and (ii) occurs in the statement of Theorem 3.2 and the equivalence of (i) and (iii) occurs in the statement of Theorem 3.1.

The equivalences of (ii) and (iv), of (iv) and (vii), and of (vii) and (viii) are immediate from Lemma 3.3, since the space $L_{w}^{1}(v)$ always has the Fatou property.

Finally, the equivalences of (iii) and (v), of (v) and (vii), and of (vii) and (ix) are also immediate from Lemma 3.3 since the space $L^{1}(v)$ always has a Lebesgue topology.

According to [12, p. 31] and [13, Theorem 5.1], condition (i) of Proposition 3.4 is satisfied whenever the Fréchet space in which $v$ takes its values does not contain an isomorphic copy of $c_{0}$.

An examination of the proof of Proposition 3.4 shows that it can be adapted to establish the following more general result. Recall that $L_{\left\{\rho_{n}\right\}}$ is super order dense in $\left(L_{\left\{\rho_{n}\right\}}\right)^{F}$ means that for every element $0 \leq u \in\left(L_{\left\{\rho_{n}\right\}}\right)^{F}$ there exists a sequence $\left(u_{k}\right)_{k} \subseteq L_{\left\{\rho_{n}\right\}}^{+}$satisfying $u_{k} \uparrow u$ in $\left(L_{\left\{\rho_{n}\right\}}\right)^{F}$ [3, Definition 1.9].

PROPOSITION 3.5. Let $L_{\left\{\rho_{n}\right\}}$ be a Fréchet function space generated by the increasing fundamental sequence of function seminorms $\left\{\rho_{n}\right\}_{n \in \mathbb{N}}$. Assume, for each $m \in \mathbb{N}$, that the restriction of $\left(\rho_{m}\right)_{L}$ to $L_{\left\{\rho_{n}\right\}}$ coincides with $\rho_{m}$, that $\left(\left(L_{\left\{\rho_{n}\right\}}\right)^{F}\right)_{a}=L_{\left\{\rho_{n}\right\}}$ and that $L_{\left\{\rho_{n}\right\}}$ is super order dense in $\left(L_{\left\{\rho_{n}\right\}}\right) F$. Then the following assertions are equivalent.

(i) $L_{\left\{\rho_{n}\right\}}=\left(L_{\left\{\rho_{n}\right\}}\right)^{F}$.

(ii) $\left(L_{\left\{\rho_{n}\right\}}\right)^{F}$ has a $\sigma$-Lebesgue topology.

(iii) $L_{\left\{\rho_{n}\right\}}$ has the $\sigma$-Fatou property.

(iv) $\left(L_{\left\{\rho_{n}\right\}}\right)^{F}$ is a KB-space.

(v) $L_{\left\{\rho_{n}\right\}}$ is a KB-space.

(vi) $\left(L_{\left\{\rho_{n}\right\}}\right)^{F}$ contains no lattice copy of $c_{0}$.

(vii) $L_{\left\{\rho_{n}\right\}}$ contains no lattice copy of $c_{0}$.

(viii) $\left(L_{\left\{\rho_{n}\right\}}\right)^{F}$ is weakly sequentially complete.

(ix) $L_{\left\{\rho_{n}\right\}}$ is weakly sequentially complete.

We conclude with an application to function spaces induced by a Köthe matrix which was stated, without proof, in [4, p. 94].

COROLLARY 3.6. In the notation of Example 2, let $\rho$ be a determining function norm with the Fatou property. Then $L_{\left\{\rho_{n}\right\}}$ is complete and $\left\{\rho_{n}\right\}_{n \in \mathbb{N}}$ is also determining. 
PROOF. Since $\rho$ has the Fatou property, so does each $\rho_{n}$, for $n \in \mathbb{N}$, that is, $\left\{\rho_{n}\right\}_{n \in \mathbb{N}}$ has the Fatou property. By Theorem 2.4, $L_{\left\{\rho_{n}\right\}}$ is complete.

Since $\rho$ is determining, choose $u \in L_{\rho}$ that is positive $\mu$-a.e. Then the sets $A_{n}:=$ $\left\{w \in \Omega \mid u(w) \geq n^{-1}\right\}$, for all $n \in \mathbb{N}$, satisfy $A_{n} \uparrow \Omega$. Let $A \in \Sigma$ satisfy $\mu(A)>0$. Then $A \cap A_{n} \uparrow A$ and so there exists $m \in \mathbb{N}$ such that $\mu\left(A_{m} \cap A\right)>0$. Moreover, $\chi_{A \cap A_{m}} \in L_{\rho}$ because $\chi_{A_{m} \cap A} \leq \chi_{A_{m}} \leq m \cdot u$ with $m \cdot u \in L_{\rho}$. So $\rho$ is saturated [22, Ch. 15, Section 67]. It follows from [18, Lemma 1.1] that there is an increasing sequence $(\Omega(k))_{k} \subseteq \Sigma$ with $\bigcup_{k=1}^{\infty} \Omega(k)=\Omega\left(\mu\right.$-a.e.) such that $\left(\chi_{\Omega(k)}\right)_{k} \subseteq L_{\rho}$ and, for each $(k, n) \in \mathbb{N}^{2}$, there exists $\beta(k, n)>0$ satisfying $a_{n} \chi_{\Omega(k)} \leq \beta(k, n) \chi_{\Omega(k)}$ for all $(k, n) \in \mathbb{N}^{2}$, pointwise on $\Omega$. All these inequalities and the definition of $\rho_{n}$ imply that $\rho_{n}\left(\chi_{\Omega(k)}\right)<\infty$ for all $k, n \in \mathbb{N}$, that is, $\left(\chi_{\Omega(k)}\right)_{k} \subseteq L_{\left\{\rho_{n}\right\}}$. Since $L_{\left\{\rho_{n}\right\}}$ is metrizable, there exist positive numbers $\lambda_{k}$, for all $k \in \mathbb{N}$, such that $\left(\lambda_{k} \chi_{\Omega(k)}\right)_{k}$ is a bounded sequence in $L_{\left\{\rho_{n}\right\}}$. Moreover, we can choose $0<\alpha_{k} \leq \lambda_{k}$ with $\sum_{k} \alpha_{k}<\infty$. Then the series $\sum_{k} \alpha_{k} \chi \Omega(k)=v$ is absolutely summable in $L_{\left\{\rho_{n}\right\}}$. Completeness of $L_{\left\{\rho_{n}\right\}}$ ensures that $v \in L_{\left\{\rho_{n}\right\}}^{+}$, with $v>0$ ( $\mu$-a.e.) on $\Omega$. So $\left\{\rho_{n}\right\}_{n \in \mathbb{N}}$ is determining.

\section{References}

[1] A. A. Albanese, 'Primary products of Banach spaces', Arch. Math. 66 (1996), 397-405.

[2] A. A. Albanese, 'On subspaces of the spaces $L_{\mathrm{loc}}^{p}$ and of their strong duals', Math. Nachr. 197 (1999), 5-18.

[3] C. D. Aliprantis and O. Burkinshaw, Locally Solid Riesz Spaces (Academic Press, New York, 1978).

[4] J. Bonet, S. Okada and W. J. Ricker, 'The canonical spectral measure and Köthe function spaces', Quaest. Math. 29 (2006), 91-116.

[5] J. M. F. Castillo, J.C. Díaz and J. Motos, 'On the Fréchet space $L^{p-}$, Manuscripta Math. 96 (1988), 219-230.

[6] G. P. Curbera, 'Operators into $L^{1}$ of a vector measure and applications to Banach lattices', Math. Ann. 293 (1992), 317-330.

[7] G. P. Curbera and W. J. Ricker, 'Banach lattices with the Fatou property and optimal domains of kernel operators', Indag. Math. (N.S.) 17 (2006), 187-204.

[8] R. del Campo and W. J. Ricker, 'The space of scalarly integrable functions for a Fréchet spacevalued measure', J. Math. Anal. Appl. 354 (2009), 641-647.

[9] L. Drewnowski and I. Labuda, 'Copies of $c_{0}$ and $\ell_{\infty}$ in topological Riesz spaces', Trans. Amer. Math. Soc. 350 (1998), 3555-3570.

[10] D. H. Fremlin, Topological Riesz Spaces and Measure Theory (Cambridge University Press, Cambridge, 1974).

[11] N. J. Kalton, 'Exhaustive operators and vector measures', Proc. Edinb. Math. Soc. 19 (1974), 291-300.

[12] I. Kluvánek and G. Knowles, Vector Measures and Control Systems (North-Holland, Amsterdam, 1976).

[13] D. R. Lewis, 'On integrability and summability in vector spaces', Illinois J. Math. 16 (1972), 294-307.

[14] W. A. J. Luxemburg and A. C. Zaanen, Riesz Spaces I (North-Holland, Amsterdam, 1971).

[15] R. G. Meise and D. Vogt, Introduction to Functional Analysis (Clarendon Press, Oxford, 1997).

[16] G. Metafune and V. B. Moscatelli, 'On the space $\ell^{p+}=\bigcap_{q>p} \ell^{q}$ ', Math. Nachr. 147 (1990), 7-12.

[17] S. Okada and W. J. Ricker, 'Vector measures and integration in non-complete spaces', Arch. Math. 63 (1994), 344-353. 
[18] K. Reiher, 'Weighted inductive and projective limits of normed Köthe function spaces', Results Math. 13 (1988), 147-161.

[19] L. Ronglu and B. Qingying, 'Locally convex spaces containing no copy of $c_{0}$ ', J. Math. Anal. Appl. 172 (1993), 205-211.

[20] G. F. Stefansson, ' $L^{1}$ of a vector measure', Le Matematiche 48 (1993), 219-234.

[21] W. Wnuk, 'Locally solid Riesz spaces not containing $c_{0}$ ', Bull. Pol. Acad. Sci. Math. 36 (1988), 51-56.

[22] A. C. Zaanen, Integration, 2nd rev. edn (North-Holland, Interscience, Amsterdam, New York, 1967).

[23] A. C. Zaanen, Riesz Spaces II (North-Holland, Amsterdam, 1983).

R. DEL CAMPO, Dpto. Matemática Aplicada I, EUITA, Ctra. de Utrera Km. 1, E-41013 Sevilla, Spain

e-mail: rcampo@us.es

W. J. RICKER, Math.-Geogr. Fakultät, Katholische Universität Eichstätt-Ingolstadt, D-85072 Eichstätt, Germany

e-mail: werner.ricker@ku-eichstaett.de 